OPEN ACCESS

Edited by:

Lifeng Zhu,

Nanjing Normal University, China

Reviewed by:

Qi Wu,

Institute of Microbiology (CAS), China

Alinne Castro,

Dom Bosco Catholic University, Brazi

${ }^{*}$ Correspondence:

Hao Du

duhao@yfiac.cn

Qiwei We

weiqw@yfi.ac.cn

tThese authors have contributed equally to this work

Specialty section: This article was submitted to

Microbial Symbioses,

a section of the journa

Frontiers in Microbiology

Received: 02 December 2019 Accepted: 05 March 2020

Published: 21 April 2020

Citation:

Yang $H$, Leng $X$, Du H, Luo J, Wu J and Wei Q (2020) Adjusting

the Prerelease Gut Microbial

Community by Diet Training to Improve the Postrelease Fitness

of Captive-Bred Acipenser

dabryanus. Front. Microbiol. 11:488.

doi: 10.3389/fmicb.2020.00488

\section{Adjusting the Prerelease Gut Microbial Community by Diet Training to Improve the Postrelease Fitness of Captive-Bred Acipenser dabryanus}

\author{
Haile Yangt, Xiaoqian Lengt, Hao Du*, Jiang Luo, Jinping Wu and Qiwei Wei* \\ Key Laboratory of Freshwater Biodiversity Conservation, Ministry of Agriculture and Rural Affairs of China, Yangtze River \\ Fisheries Research Institute, Chinese Academy of Fishery Sciences, Wuhan, China
}

As one of the most important tool for biodiversity restoration and endangered species conservation, reintroduction has been implemented worldwide. In reintroduction projects, prerelease conditioning could effectively increase postrelease fitness and survival by improving animals' adaptation to transformation from artificial to natural environments. However, how early-life diet training affects individuals' adaptation, fitness, and survival after release remains largely unknown. We hypothesized that earlylife diet training would adjust the host's gut microbial community, the gut microbial community would influence the host's diet preference, and the host's diet preference would impact its adaptation to diet provision transformation and then determine postrelease fitness and survival. To verify this hypothesis, we investigated the growth characteristics and gut microbes of Yangtze sturgeon (Acipenser dabryanus) trained with natural and formula diets at both the prerelease and postrelease stages. The results showed that (1) the gut microbial communities of the individuals trained with a natural diet (i.e., natural diet group) and formula diet (i.e., formula diet group) evolved to the optimal status for their corresponding diet provisions, (2) the individuals in the natural diet group paid a lower cost (i.e., changed their gut microbial communities less) during diet transformation and release into the natural environment than did the individuals in the formula diet group, and (3) the gut microbes in the natural diet group better supported postrelease fitness and survival than did the gut microbes in the formula diet group. The results indicated that better prerelease diet training with more appropriate training diets and times could improve the reintroduction of Yangtze sturgeon by adjusting the prerelease gut microbial community. Because a relationship between diet (preference) and gut microbes is common in animals from insects (such as Drosophila melanogaster) to mammals (such as Homo sapiens), our hypothesis verified by the case study on Yangtze sturgeon applies to other animals. We therefore encourage future studies to identify optimal training diets and times for each species to best adjust its prerelease gut microbial community and then improve its postrelease fitness and survival in reintroduction projects.

Keywords: reintroduction, captive-bred population, release cost, diet training, gut microbes, Acipenser dabryanus 


\section{INTRODUCTION}

With increasing human activities, biodiversity has decreased globally (Butchart et al., 2010; Pimm et al., 2014), which has impacted Earth's ecosystems and human well-being (Cardinale et al., 2012; Hooper et al., 2012; Oliver et al., 2015). Currently, actions to restore biodiversity are being implemented globally (Mace et al., 2018). Reintroduction, which is the translocation of individuals to areas in which a species has been extirpated with the aim of re-establishing a selfsustaining population, has become a globally important form of conservation management used to restore biodiversity (CochranBiederman et al., 2015; Brichieri-Colombi and Moehrenschlager, 2016; Taylor et al., 2017; Haase and Pilotto, 2019; Jourdan et al., 2019). Reintroduction projects have been frequently conducted for many taxa, including mammals, birds, reptiles, fish, and invertebrates (Tavecchia et al., 2009; Dincă et al., 2018; Yang et al., 2018; Brichieri-Colombi et al., 2019; Jourdan et al., 2019; Teitelbaum et al., 2019). To date, over 1200 species have been reintroduced (Brichieri-Colombi et al., 2019).

Reintroduction projects require viable source populations for release, derived from either wild or captive-bred populations (Brichieri-Colombi et al., 2019). However, declines in abundance and occurrence have rendered remaining wild populations too fragile to act as continuous sources (Todd and Lintermans, 2015; Brichieri-Colombi et al., 2019), so captive-bred populations are the only choice for reintroduction projects. Captive breeding provides assurance against species extinction and an increased ability to target a specific sex or age cohort for release (International Union for Conservation of Nature [IUCN] and Species Survival Commission [SSC]., 2013; BrichieriColombi et al., 2019). However, captive-bred populations often have a relatively low postrelease success in terms of survival, behavior, or breeding performance (Letty et al., 2007; Todd and Lintermans, 2015; Brichieri-Colombi et al., 2019). Short-term postrelease (varying from a few weeks to 1 year in different long-lived species) survival is the crucial first step for the success of reintroduction projects (Svåsand et al., 2000; Armstrong and Seddon, 2008; Tavecchia et al., 2009; Bertolero et al., 2018; Cayuela et al., 2019). Prerelease conditioning has been proven to generally effectively improve short-term postrelease survival (Batson et al., 2015; Tetzlaff et al., 2019). However, starvation is still a significant source of mortality in captive carnivores post release, and foraging deficiencies are underrepresented in the literature (Berger Tal et al., 2019).

Training captive-bred prerelease animals with natural diets could result in increased postrelease survival (Whiteside et al., 2015). Whiteside et al. (2015), indicated that diet conditioning increased postrelease survival by altering foraging efficiency, food discrimination, handling skills, and gut morphology. However, diet preference underlies these four functional effects. Diet preference depends on host gut microbes, which modify host sensory perception (Wong et al., 2017; Yuval, 2017). Therefore, we assume that (i) diet training in captive-bred prerelease animals will determine the drift of host gut microbes, (ii) gut microbes will influence host diet preference, and (iii) diet preference will influence foraging behavior and then impact individuals' fitness and survival after release.

Yangtze sturgeon (Acipenser dabryanus) is a critically endangered endemic species and a flagship species in the Yangtze River (Wu et al., 2014). Because of changes in hydrological conditions driven by human activities, the Yangtze sturgeon has not reproduced naturally since 2000 (Zhang et al., 2011; Wu et al., 2014), and there is currently no natural population in the Yangtze River. Releasing captive-bred populations into appropriate reaches is the only way to restore the natural Yangtze sturgeon population. We retained the F1-F3 generations of a captive-bred population of Yangtze sturgeon and reintroduced more than 100,000 adults and juveniles into the upper Yangtze River from 2007 to 2019. Now, to test our hypothesis and improve our reintroduction programs, we experimentally manipulated early-life diets in the first 7 months after hatching by using natural diets and formula diets for two experimental groups of Yangtze sturgeon and then released some labeled individuals from these two groups into the natural environment. We monitored their body length and weight and gut microbes, and then, by analyzing the two groups' growth characteristics and gut microbes, we verified the mechanisms by which diet training impacts postrelease fitness and survival.

\section{MATERIALS AND METHODS}

\section{Experimental Design and Sampling Procedures}

The experimental fish were the F2-generation offspring of a captive-bred population of Yangtze sturgeon. Artificial reproduction of the fish was implemented at facilities of the Yangtze River Fishery Research Institute located in Jingzhou, Hubei Province, China. The offspring came from a single mating pair. A total of 1200 larvae were randomly screened and divided into two groups. Each group was split into three tanks, with 200 fish per tank. The larvae were fed earthworms beginning 8 days post hatching (dph), when the embryonic yolk was completely absorbed. Before feeding, the earthworms were soaked in 2$5 \mathrm{ppm}$ potassium permanganate for 3-5 min, irradiated with UV light for $0.5 \mathrm{~h}$ and then minced to an appropriate size for the larvae. When the larvae grew to a full length of 25$35 \mathrm{~mm}$ (approximately $23 \mathrm{dph}$ ), one group (i.e., natural diet group) continued to feed on earthworms, while the other group (i.e., formula diet group) was gradually transitioned to an artificial formula diet. The duration of the transition period from earthworms to the artificial formula diet was approximately 15 days. Under laboratory rearing conditions, the two treatment groups were fed four times daily (07:00, 13:00, 19:00, and 0:00), fully aerated tap water was used for circulation, and the water temperature and dissolved oxygen content were controlled at $19 \pm 0.5^{\circ} \mathrm{C}$ and $6.5-8.5 \mathrm{mg} / \mathrm{L}$, respectively. Because captive-bred juveniles of Yangtze sturgeon were released in the same year as part of the reintroduction project, 60 individuals of each group were selected randomly, marked, and then released into a natural pond at 7 months post hatching $(\mathrm{mph})$ and recaptured 2 months later (i.e., at $9 \mathrm{mph}$ ). 
We randomly sampled individuals from each group five times: larval stage ( $1 \mathrm{mph}$ ), early juvenile stage (2 and $3 \mathrm{mph}$ ), and juvenile stage ( 7 and $9 \mathrm{mph}$ ). At the larval stage, the formula diet group was transitioned from earthworms to the formula diet. We measured the body length and weight of the sampled individuals. All sampled individuals were euthanized with an overdose of MS 222 (Sigma, Germany) before dissection, and then, approximately $0.5 \mathrm{~g}$ of the hindgut contents was extracted aseptically. The contents from multiple individuals were pooled into one sample, especially for the small individual samples. At least three replicates per group were collected at each sampling time, and the samples were flash-frozen in liquid nitrogen and then stored at $-80^{\circ} \mathrm{C}$ until DNA extraction. The experimental procedure is conceptually outlined in Figure 1.

\section{DNA Extraction and Sequence Analysis}

Microbial DNA was extracted from gut samples using an E.Z.N.A. ${ }^{\circledR}$ Stool DNA Kit (Omega BioTek, Norcross, GA, United States) according to the manufacturer's protocols. Then, the final DNA concentration and purity were determined by a NanoDrop 2000 UV-vis spectrophotometer (Thermo Fisher Scientific, Wilmington, United States), and DNA quality was checked by $1 \%$ agarose gel electrophoresis. The V3-V4 hypervariable regions of the bacterial 16S rRNA gene were amplified with the primers 338F (5'-ACTCCTACGGGAGGCAGCAG-3') and 806R $\left(5^{\prime}\right.$-GGACTACHVGGGTWTCTAAT- $\left.3^{\prime}\right)$ by using a PCR thermocycler system (GeneAmp 9700, ABI, United States). The PCRs were conducted using the following program: $3 \mathrm{~min}$ of denaturation at $95^{\circ} \mathrm{C} ; 27$ cycles of $30 \mathrm{~s}$ at $95^{\circ} \mathrm{C}, 30 \mathrm{~s}$ for annealing at $55^{\circ} \mathrm{C}$, and $45 \mathrm{~s}$ for elongation at $72^{\circ} \mathrm{C}$; and a final extension at $72^{\circ} \mathrm{C}$ for $10 \mathrm{~min}$. PCRs were performed in triplicate $20 \mu \mathrm{l}$ mixtures containing $4 \mu \mathrm{l}$ of $5 \times$ FastPfu Buffer, $2 \mu \mathrm{l}$ of $2.5 \mathrm{mM}$ dNTPs, $0.8 \mu \mathrm{l}$ of each primer $(5 \mu \mathrm{M}), 0.4 \mu \mathrm{l}$ of FastPfu Polymerase, and $10 \mathrm{ng}$ of template DNA. The resulting PCR products were extracted from a $2 \%$ agarose gel, further purified using an AxyPrep DNA Gel Extraction Kit (Axygen Biosciences, Union City, CA, United States) and quantified using QuantiFluor ${ }^{\mathrm{TM}}$-ST (Promega, United States) according to the manufacturer's protocol.

Purified amplicons were pooled in equimolar amounts and subjected to paired-end sequencing $(2 \times 300 \mathrm{bp})$ on an Illumina MiSeq platform (Illumina, San Diego, CA, United States) according to standard protocols by Majorbio Bio-Pharm Technology Co., Ltd. (Shanghai, China). Raw fastq files were demultiplexed, quality-filtered by Trimmomatic, and merged by FLASH. Operational taxonomic units (OTUs) were clustered

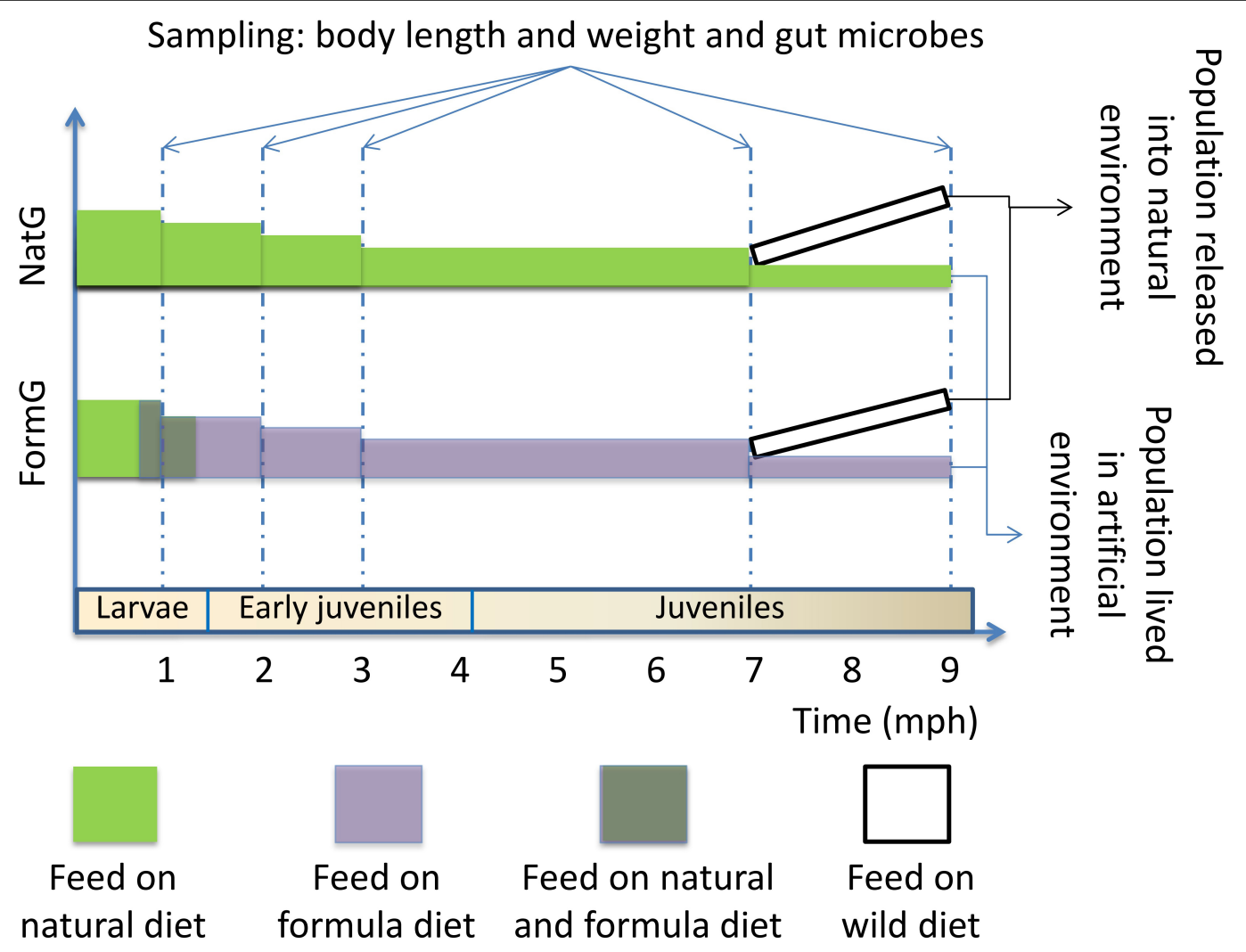

FIGURE 1 | Conceptual diagram showing the experimental procedure. The sampling times and corresponding developmental stages are shown. At 1 mph, the formula diet group was undergoing diet transformation. At $7 \mathrm{mph}$, some individuals from the two groups were released into the natural environment; the individuals were recaptured 2 months later ( 9 mph). Sampling includes measurement of body length and weight and extraction of hindgut contents. NatG, natural diet group; FormG, formula diet group; mph, months post hatching. 
with a $97 \%$ similarity cutoff using UPARSE (version 7.1$)^{1}$, and chimeric sequences were identified and removed using UCHIME. The taxonomy of each 16S rRNA gene sequence was analyzed by the RDP Classifier algorithm ${ }^{2}$ against the Silva (SSU128) $16 \mathrm{~S}$ rRNA database using a confidence threshold of $70 \%$. The raw data have been deposited in the $\mathrm{CNSA}^{3}$ of $\mathrm{CNGBdb}$ with accession number CNP0000907.

\section{Statistical Analysis}

The samples of both groups, including individuals living in the artificial environment and recaptured individuals, from five stages $(1,2,3,7$, and $9 \mathrm{mph}$ ) were delineated into 10 subgroups. Alpha diversity analysis, including analysis of the Chao richness index, Shannon diversity index, and Simpson diversity index, was conducted to reveal the variation in all gut microbial samples. Beta diversity analysis, including hierarchical clustering, sample distances, and non-metric multidimensional scaling (NMDS) analysis, was conducted to reveal the similarity of samples based on their gut microbial communities. The gut microbial community of each subgroup was analyzed at the family level. Analysis of the common OTUs, species, genera, and families between adjacent developmental stages of each group was performed to examine the drift of gut microbial communities with diet training by the natural and formula diets. Analysis of the common OTUs, species, genera, and families between the two groups at each developmental stage was performed to examine the gut microbial community divergence between the natural diet group and the formula diet group. Analysis of the common OTUs, species, genera, and families between each stage and the recaptured stage of each group was performed to examine the fitness of gut microbial communities for release. Then, based on these data sets, (1) lines of best fit for fitness at the OTU, species, genus, and family levels were generated for each group using a polynomial fitting method, and (2) the drift of gut microbial communities was outlined.

\section{RESULTS}

\section{Growth Characteristics}

The growth characteristics of the two groups are shown in Table 1. The body length and weight of the Yangtze sturgeon in the two groups increased continuously from 1 to $9 \mathrm{mph}$ in the artificial environment. The growth characteristics of the individuals in the two groups did not show a significant difference at $1 \mathrm{mph}$. However, the growth characteristics of the formula diet group at the early juvenile stage (2 and $3 \mathrm{mph}$ ) were significantly lower than that of the natural diet group after the diet switch began $(P<0.01$ and $P<0.05)$. Then, these significant differences vanished as the individuals of the formula diet group adapted to the formula diet at the juvenile stage (7 and $9 \mathrm{mph}$ ) $(P>0.05)$. After living in the natural environment for two months $(9 \mathrm{mph})$, the recaptured individuals of both groups

\footnotetext{
${ }^{1}$ http://drive5.com/uparse/

${ }^{2}$ http://rdp.cme.msu.edu/

${ }^{3}$ https://db.cngb.org/cnsa/
}

exhibited significantly lower growth characteristics than the individuals living in the artificial environment. The recaptured individuals of the natural diet group showed significantly higher growth characteristics than the recaptured individuals of the formula diet group $(P<0.05)$.

Moreover, after 2 months of 120 individuals being released into a natural pond, 37 individuals with natural diet group labels and 32 individuals with formula diet group labels were recaptured. The recapture rate of the natural diet group was $61.67 \%$, and that of the formula diet group was $53.33 \%$. In other words, the survival rate of the natural diet group was $61.67 \%$, and that of the formula diet group was $53.33 \%$.

\section{Gut Microbes}

A total of 1,406,433 clean sequences were obtained from 38 samples of the two groups, and the average length of these sequences was $437.70 \mathrm{bp}$. A total of 2165 kinds of bacterial OTUs were detected (UPARSE, 97\% cutoff) among these sequences, which belonged to 39 phyla, 84 classes, 192 orders, 351 families, 738 genera, and 1202 species (more details in Supporting Information 1_OTU Table). The OTU counts and compositions of each subgroup were highly variable among sampling times (Figure 2, more details in Supporting Information 2_Subgroup Delineation and Supporting Information 3_Alpha Diversity and Beta Diversity). The dominant taxa at the family level were Clostridiaceae, Enterobacteriaceae, Fusobacteriaceae, Streptococcaceae, Flavobacteriaceae, and Moraxellaceae (more details are provided in Supporting Information 4_Community Heatmap).

\section{Gut Microbial Community Drift}

Along with the types of OTUs, the species, genera, and families in the gut microbial communities declined from 1 to 2 to $3 \mathrm{mph}$ (Figure 2), and the contribution rates (indicated by coverage) from the former stage to the latter stage increased in the natural diet group but decreased in the formula diet group (Figure 3). Along with the types of OTUs, the species, genera, and families in the gut microbial communities increased from 3 to 7 to 9 mph (Figure 2), with the contribution rates from the former stage to the latter stage decreasing and then increasing in the natural diet group but continually decreasing in the formula diet group (Figure 3).

The divergence of gut microbial communities between the natural diet group and the formula diet group increased continually from 1 to $7 \mathrm{mph}$ in the artificial environment (Figure 4). However, the divergence in the natural environment was obviously weaker than that in the artificial environment (Figure 4). In other words, the divergence trend between the two groups was reversed after the individuals were released into the natural environment.

The fitness (indicated by coverage) of the gut microbial communities for release in both the natural and formula diet groups declined continually from 1 to $3 \mathrm{mph}$, followed by some recovery at $7 \mathrm{mph}$ (Figure 5). The fitness declined more sharply and recovered more weakly in the formula diet group than in the natural diet group (Figure 5). The lines of best fit showed that the fitness of gut microbial communities for release would continue 
TABLE 1 | Growth characteristics of the natural diet group and formula diet group of Acipenser dabryanus.

\begin{tabular}{|c|c|c|c|c|c|c|c|}
\hline & & \multirow{2}{*}{$\begin{array}{l}\text { Larval stage } \\
\qquad 1 \mathrm{mph}\end{array}$} & \multicolumn{2}{|c|}{ Early juvenile stage } & \multicolumn{2}{|c|}{ Juvenile stage } & \multirow{2}{*}{$\begin{array}{c}\text { Juvenile stage (recaptured) } \\
9 \mathrm{mph}\end{array}$} \\
\hline & & & $2 \mathrm{mph}$ & $3 \mathrm{mph}$ & $7 \mathrm{mph}$ & $9 \mathrm{mph}$ & \\
\hline & FormG & $2.07 \pm 0.13$ & $5.47 \pm 0.63$ & $11.72 \pm 0.89$ & $23.49 \pm 2.72$ & $29.28 \pm 1.44$ & $23.89 \pm 2.74$ \\
\hline & $P$-value & $>0.05$ & $<0.01^{\star \star}$ & $<0.01^{\star \star}$ & $>0.05$ & $>0.05$ & $<0.05^{\star}$ \\
\hline Body weight (g) & NatG & $0.08 \pm 0.02$ & $2.07 \pm 0.56$ & $20.10 \pm 3.39$ & $80.22 \pm 20.85$ & $152.00 \pm 23.62$ & $105.27 \pm 27.20$ \\
\hline
\end{tabular}

Significance is indicated by ${ }^{*} P<0.05$ and ${ }^{* *} P<0.01$. NatG, natural diet group; FormG, formula diet group; mph, months post hatching.

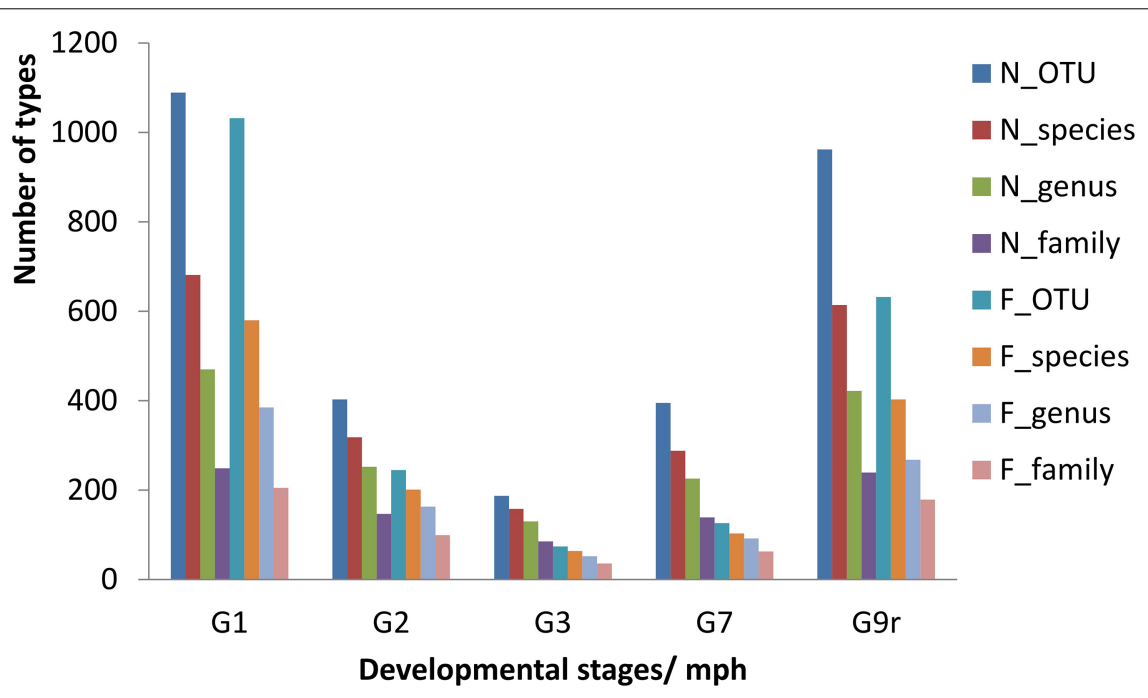

FIGURE 2 | Taxonomic characteristics of gut microbes of the natural diet group and formula diet group of Acipenser dabryanus at different stages. G1: the sample subgroup sampled at $1 \mathrm{mph}$; G2: the sample subgroup sampled at $2 \mathrm{mph}$; G9r: the sample subgroup sampled from recaptured individuals at 9 mph; N_OTU: natural diet group at the OTU level; F_family: formula diet group at the family level; mph: month post hatching.

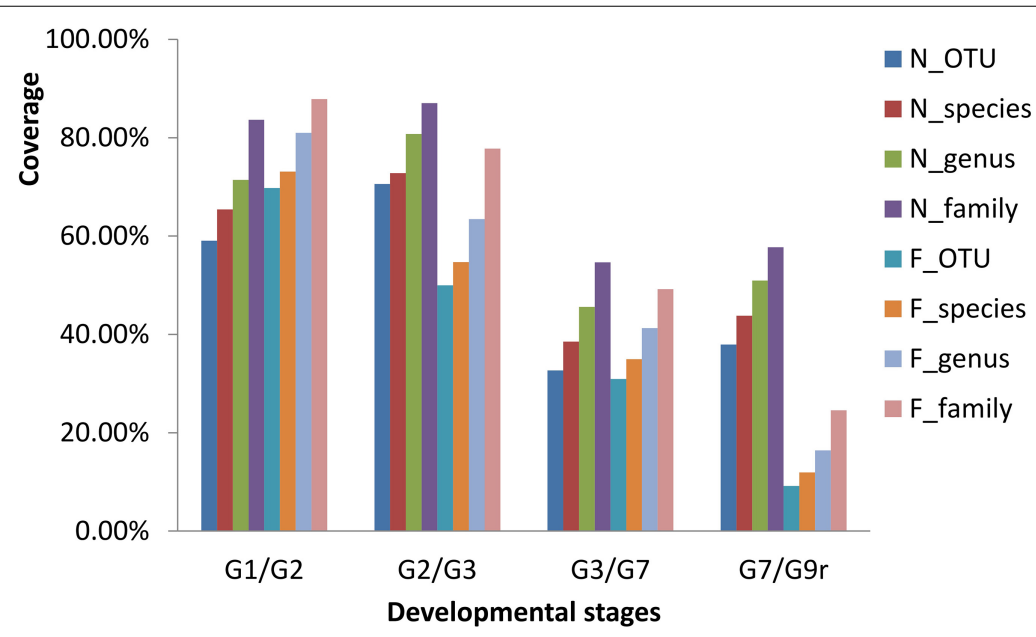

FIGURE 3 | Drift of the gut microbial communities of Acipenser dabryanus in the natural diet group and formula diet group. Coverage is the proportion of common OTUs, species, genera and families in two adjacent stages (such as G1 and G2) compared to the later stage (G2). G1/G2: the drift of the gut microbial communities from $1 \mathrm{mph}$ to $2 \mathrm{mph}$; G2/G3: the drift of the gut microbial communities from $2 \mathrm{mph}$ to $3 \mathrm{mph}$; G7/G9r: the drift of the gut microbial communities from $7 \mathrm{mph}$ to 9 mph (recaptured); N_OTU: natural diet group at the OTU level; F_family: formula diet group at the family level; mph: month post hatching. 


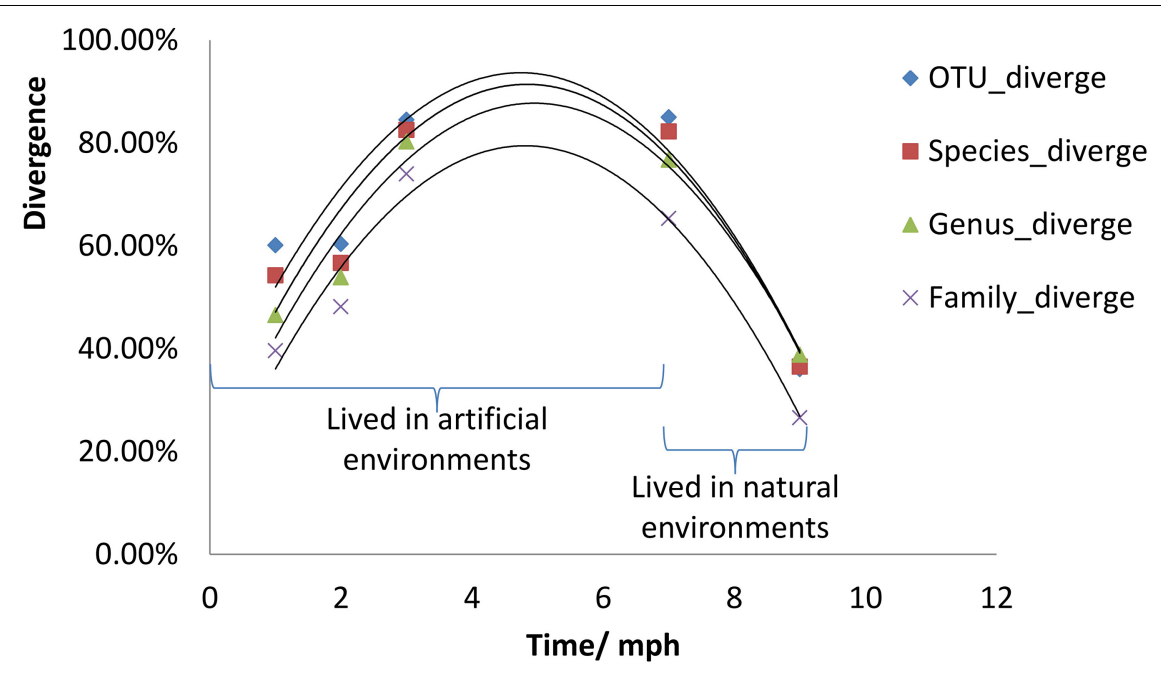

FIGURE 4 | Divergence of gut microbial communities of Acipenser dabryanus between the natural diet group and the formula diet group. Divergence is the proportion of total OTUs, species, genera or families unshared by two groups (natural diet group and formula diet group). OTU_diverge: divergence at the OTU level; Species_diverge: divergence at the Species level; mph: month post hatching.

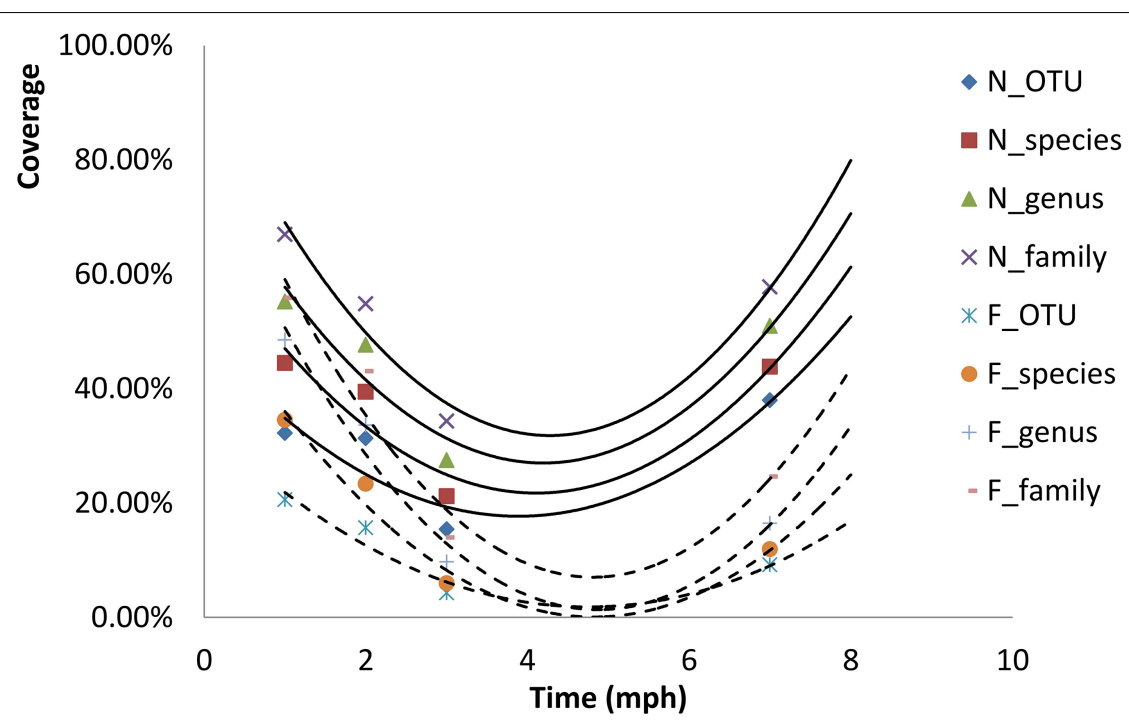

FIGURE 5 | Fitness (coverage) of gut microbial communities of each group of Acipenser dabryanus at the OTU, species, genus and family levels at different developmental stages and their lines of best fit. N_OTU: natural diet group at the OTU level; F_family: formula diet group at the family level; mph: month post hatching.

to recover after 7 months of natural diet training following Yangtze sturgeon hatching (Figure 5).

The continually increasing divergence of gut microbial communities between the natural diet group and the formula diet group from 1 to $7 \mathrm{mph}$ in the artificial environment (Figure 4) indicated that the gut microbial communities of the two groups evolved to different statuses. The decreased divergence of gut microbial communities between the natural diet group and the formula diet group at $9 \mathrm{mph}$ in the natural environment (Figure 4) indicated that the gut microbial communities of the two groups began to converge to a common optimal status that fit the natural environment. Because the fitness of the gut microbial community for release in the formula diet group was lower than that in the natural diet group (Figure 5), the optimal status of the gut microbial community in the natural environment was more similar to that of the natural diet group than to that of the formula diet group (Figure 6).

\section{DISCUSSION}

\section{Diet Training Adjusts Gut Microbes}

Diet training changed the gut microbial communities of Yangtze sturgeon individuals. It is well known that diet impacts the types 


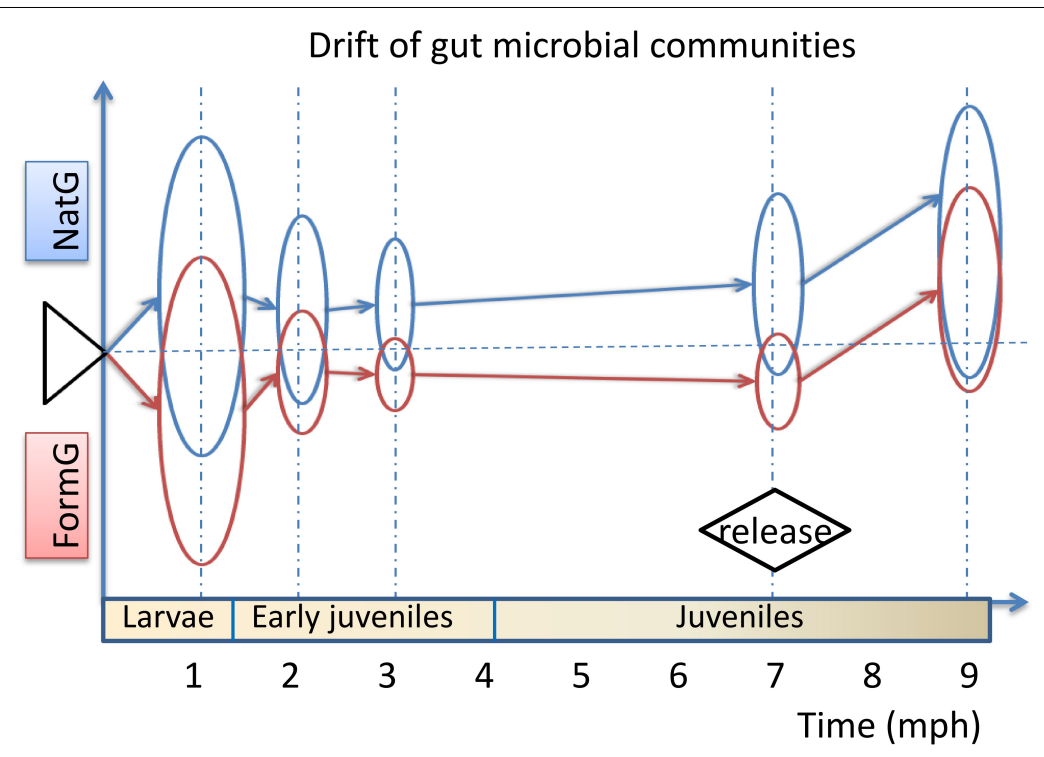

FIGURE 6 | Conceptual diagram showing the drift of gut microbial communities of Acipenser dabryanus in the natural diet group and formula diet group at different developmental stages. The ovals represent gut microbial communities. The size of the oval indicates the types of OTU in the gut microbial community of each subgroup. The location of the oval indicates the composition of the gut microbial community of each subgroup. NatG: natural diet group; FormG: formula diet group; mph: months post hatching.

of microorganisms that colonize the gut (Smith et al., 2015; Gajardo et al., 2017; Yang et al., 2019). Generally, the bacterial richness and diversity of developing larvae significantly increase during the first hours, days, or weeks post hatching and then remain stable (Califano et al., 2017; Li et al., 2017; WilkesWalburn et al., 2019). As diet is a major contributor to early microbial community development, diet transformation leads to obvious changes in the gut microbial community (WilkesWalburn et al., 2019). In the present study, from 1 to $7 \mathrm{mph}$, the composition of gut microbes varied obviously (Figure 2 and Supplementary Figures SI3_1, 2, 3), and only some microbial types survived to the subsequent stages in both groups (Figure 3 ), which indicated that the gut microbial communities in each group were drifting. Along with the drift of gut microbial communities, the common microbial types in both groups decreased from 1 to $7 \mathrm{mph}$ (Figure 4), which indicated that the diet training difference drove the gut microbial communities to drift toward different assemblages.

\section{Gut Microbes Strengthen Diet Preference}

Drift of the gut microbial communities influenced Yangtze sturgeons' preference for a natural diet or formula diet. Gut microbes can impact hosts' diet preferences and then shape host fitness-related behavior (Alcock et al., 2014; Wong et al., 2017; Akami et al., 2019; Murgier et al., 2019; Patnode et al., 2019). In the current study, at $1 \mathrm{mph}$, the natural diet group was fed earthworms, and the formula diet group began to transition from earthworms to a formula diet. The mismatch between diet provision and diet preference driven by gut microbes was not very strong, so there were similar growth characteristics between he two groups at $1 \mathrm{mph}$ (Table 1). At 2 and $3 \mathrm{mph}$, the formula diet group was fed only the formula diet, and the gut microbial communities had not evolved to an optimal assemblage matching the new diet. The mismatch between diet preference and diet provision was substantial, which caused the formula diet group to have significantly lower growth characteristics than the natural diet group (Table 1). After more than 5 months of adjustment, along with the gut microbial communities of the formula diet group evolving to the optimal assemblage for the formula diet, at 7 and $9 \mathrm{mph}$, the individuals of the formula diet group displayed better growth efficiency, and the previously significant difference in growth characteristics between the natural diet group and formula diet group vanished (Table 1). In other words, under training with different diets, the gut microbial communities in the two groups drifted to different assemblages that supported different diet preferences and matched the different diet provisions. This finding indicated that the gut microbial community impacted diet preference, the mismatch between gut microbial communities and diet provision hampered individuals' fitness, and the adjustment between gut microbial communities and diet provision supported individuals' fitness. This finding provides a framework for understanding why $30-40 \%$ of the adult individuals of the natural diet group starved to death when fed a formula diet (Du, 2014).

\section{Prerelease Diet Preference Impacts Postrelease Fitness}

Prerelease diet preference impacted the postrelease survival rates and fitness of Yangtze sturgeon. After more than five months of adjustment to diet transformation, at $7 \mathrm{mph}$, there was no significant difference in growth characteristics between the natural diet group and the formula diet group (Table 1), which indicated that the individuals of the natural diet group were 
suited to the natural diet and that the individuals of the formula diet group were suited to the formula diet. As the diets provided in the natural environment were more similar to the natural diet than to the formula diet, the individuals in the natural diet group had a higher survival rate and better growth characteristics after release than did those in the formula diet group (Table 1). At the same time, as the diet provisions in the natural environment was different from that in the artificial environment, the released individuals had lower growth characteristics than the artificially fed individuals in both groups (Table 1). This result indicated that the mismatch between prerelease diet preference and postrelease diet provision caused low individual fitness, with greater mismatch causing lower fitness. This is why the survival of reintroduced adults was lower than the survival of adults in the source and control populations and the F1-generation offspring of reintroduced animals survived at rates similar to those of individuals in the source and control populations (Cayuela et al., 2019). The mismatch between the gut microbial community and diet provision was strong enough to have an effect in reintroduced adults but weak enough to go unnoticed in the F1-generation offspring of reintroduced animals.

\section{The Natural Diet Group Paid a Lower Cost to Adjust Their Gut Microbes to the Natural Environment}

To adapt to the natural environment, the formula diet group paid a higher cost for transforming the prerelease gut microbial community into a new optimal assemblage than did the natural diet group. As the gut microbial community impacted host diet preference, to match the diet provision in the natural environment, the gut microbial communities of released individuals needed to gradually evolve into a new optimal assemblage from the prerelease gut microbial community. Because the natural diet was more similar to the diet provision in the natural environment than was the formula diet, the similarity (coverage) of gut microbial communities between prerelease individuals and recaptured surviving individuals was higher in the natural diet group than in the formula diet group (Figure 5). This result indicated that the natural diet group more easily adapted to the environmental change caused by release than did the formula diet group.

\section{Improvement in Diet Training Depends on the Diet Chosen and Training Time}

Better diet training is possible if better diets are chosen with suitable training times. Because the diet provisions in the artificial environment and the natural environment were different, after being released into the natural environment, all individuals needed to adjust their gut microbial communities and diet preferences in order to adapt to the new diet provision. Then, there was an obvious difference in the gut microbial community between prerelease individuals and recaptured individuals (Figure 5 and Supplementary Figures SI3_1, 2, 3). The postrelease mortality rates were $38.33 \%$ (in the natural diet group) and $46.67 \%$ (in the formula diet group). The growth characteristics of the individuals living in the artificial environment were better than those of the recaptured individuals that had survived post release (Table 1). Using diets that are similar to the diet provision in natural environments to train individuals before release and construct suitable gut microbial communities matching natural environments would be helpful. Because natural populations of juvenile Yangtze sturgeon feed on aquatic oligochaetes, aquatic dipteran larvae, cladocerans, and copepods, and adult Yangtze sturgeon feed on benthic invertebrates and small fishes (Du, 2014), the diet assemblages for training Yangtze sturgeon individuals before release should be more diverse than earthworms. Moreover, a suitable training time that could allow the formation of suitable gut microbial communities is also valuable. For Yangtze sturgeon, because the results showed that the fitness (coverage) of gut microbial communities for release was appropriately 50\% after 7 months of natural diet training and would continue to increase after 7 months of natural diet training (Figure 5), we suggest that more than 7 months of natural diet training after hatching would be suitable.

\section{Improvement in the Reintroduction Effect Requires Improvement in the Source Population With Diet Training}

To improve the reintroduction effect, a viable optimal source population is needed. As approximately one-third of postrelease mortality takes place within the first month after release (Tavecchia et al., 2009), short-term postrelease survival is crucial for the success of reintroduction projects (Armstrong and Seddon, 2008; Bertolero et al., 2018; Cayuela et al., 2019). Starvation has been implicated as a significant source of mortality in the first weeks to months in reintroduced captive carnivores (Jule et al., 2008; Kemp and Roshier, 2016; Berger Tal et al., 2019). Better diet training could improve reintroduced individuals' foraging behavior and thereby improve short-term postrelease survival (Whiteside et al., 2015; Berger Tal et al., 2019). As diet training can adjust hosts' gut microbes, impact hosts' diet preference, and then influence short-term postrelease survival rates, for better reintroduction effects, improved source populations with improved diet training are needed. Improved diet training requires diets that are similar to the diet provision in the natural ecosystem and a suitable training time. For Yangtze sturgeon, although prerelease individuals of the natural diet group exhibited high similarity to recaptured individuals at both 1 and $7 \mathrm{mph}$ (Figure 5), we suggest that more than 7 months of natural diet training after hatching is more suitable for release, as populations with larger individuals exhibit a higher survival rate after reintroduction into the natural environment (Sarrazin and Legendre, 2000; Svåsand et al., 2000). Because a relationship between diet (preference) and gut microbes is common in animals from insects (such as Drosophila melanogaster) to mammals (such as Homo sapiens) (Wong et al., 2017; Akami et al., 2019; Liu et al., 2019; Murgier et al., 2019; Patnode et al., 2019), our hypothesis applies to other animals. Therefore, we encourage further experiments aiming to formulate improved diet training protocols with optimal diets and training times for reintroduction projects of other animals. 


\section{CONCLUSION}

Starvation is a significant source of mortality in captive-bred carnivores after release, and prerelease diet training could improve short-term postrelease survival. Understanding the mechanism of diet training effects is crucial for successful reintroduction projects. In this manuscript, we explore the mechanism by which diet training influences short-term postrelease fitness and survival. Diet training adjusts host gut microbes, which then impact host diet preference. Prerelease diet preference impacts host postrelease fitness, which then impacts postrelease survival rates. Thus, we propose that better diet training leads to a more suitable gut microbial community for release, followed by better postrelease fitness and ultimately better reintroduction effects. Better diet training of a prerelease source population requires (1) the use of better diet assemblages that are similar to the diet provision in natural environments and (2) a more appropriate training time that is long enough to ensure that the host gut microbes form a suitable assemblage for the natural environment.

\section{DATA AVAILABILITY STATEMENT}

The datasets generated for this study can be found in the CNSA (https://db.cngb.org/cnsa/) of CNGBdb with accession number CNP0000907.

\section{ETHICS STATEMENT}

The animal study was conscientiously abide by the ethical principles of animal welfare in People's Republic of China and Hubei Province, accepted the supervision and inspection of the Animal Experimental Ethical Committee of Laboratory Animal Centre, Yangtze River Fisheries Research Institute, Chinese Academy of Fishery Sciences.

\section{AUTHOR CONTRIBUTIONS}

HY contributed to the conceptualization, formal analysis, visualization, writing the original draft, and reviewing and editing

\section{REFERENCES}

Akami, M., Andongma, A. A., Zhengzhong, C., Nan, J., Khaeso, K., Jurkevitch, E., et al. (2019). Intestinal bacteria modulate the foraging behavior of the oriental fruit fly Bactrocera dorsalis (Diptera: Tephritidae). PLoS ONE 14:e210109. doi: 10.1371/journal.pone.0210109

Alcock, J., Maley, C. C., and Aktipis, C. A. (2014). Is eating behavior manipulated by the gastrointestinal microbiota? Evolutionary pressures and potential mechanisms. BioEssays 36, 940-949.

Armstrong, D. P., and Seddon, P. J. (2008). Directions in reintroduction biology. Trends Ecol. Evol. 23, 20-25.

Batson, W. G., Gordon, I. J., Fletcher, D. B., Manning, A. D., and Hayward, M. (2015). REVIEW: translocation tactics: a framework to support the IUCN Guidelines for wildlife translocations and improve the quality of applied methods. J. Appl. Ecol. 52, 1598-1607. the manuscript. XL worked on the methodology, investigation, data curation, formal analysis, conceptualization, funding acquisition, project administration, supervision, validation, and reviewing and editing the manuscript. HD was responsible for the conceptualization, funding acquisition, project administration, supervision, validation, and reviewing and editing the manuscript. JL and JW carried out the investigation. QW was responsible for the project administration, resources, supervision, and validation.

\section{FUNDING}

This work was supported by the National Natural Science Foundation of China (Grant No. 31772854), the National Agriculture Public Welfare Industry Research Project (Grant No. 20090304802), the Central Public-Interest Scientific Institution Basal Research Fund (Grant No. 2017HY-ZD0405, 2020TD08), and the Ocean Park Conservation Foundation of Hong Kong (Grant No. 4040000090).

\section{ACKNOWLEDGMENTS}

The data are analyzed on the online platform of Majorbio Cloud Platform (www.majorbio.com).

\section{SUPPLEMENTARY MATERIAL}

The Supplementary Material for this article can be found online at: https://www.frontiersin.org/articles/10.3389/fmicb. 2020.00488/full\#supplementary-material

DATA SHEET S1 | OTU Table showed the details of gut microbial composition of all samples.

DATA SHEET S2 | Subgroup Delineation showed the gut microbial communities and the details of redelineating subgroup.

DATA SHEET S3 | Alpha Diversity and Beta Diversity showed the Alpha diversity and Beta diversity of the gut microbial communities of all samples.

DATA SHEET S4 | Community Heatmap showed the 50 most abundant families in bacterial communities of each subgroup.

Berger Tal, O., Blumstein, D. T., and Swaisgood, R. R. (2019). Conservation translocations: a review of common difficulties and promising directions. Anim. Conserv. doi: 10.1111/acv.12534

Bertolero, A., Pretus, J. L., and Oro, D. (2018). The importance of including survival release costs when assessing viability in reptile translocations. Biol. Conserv. 217, 311-320.

Brichieri-Colombi, T. A., Lloyd, N. A., McPherson, J. M., and Moehrenschlager, A. (2019). Limited contributions of released animals from zoos to North American conservation translocations. Conserv. Biol. 33, 33-39.

Brichieri-Colombi, T. A., and Moehrenschlager, A. (2016). Alignment of threat, effort, and perceived success in North American conservation translocations. Conserv. Biol. 30, 1159-1172.

Butchart, S. H. M., Walpole, M., Collen, B., van Strien, A., Scharlemann, J. P. W., Almond, R. E. A., et al. (2010). Global biodiversity: Indicators of recent declines. Science 328, 1164-1168. doi: 10.1126/science.1187512 
Califano, G., Castanho, S., Soares, F., Ribeiro, L., Cox, C. J., Mata, L., et al. (2017). Molecular taxonomic profiling of bacterial communities in a gilthead seabream (Sparus aurata) hatchery. Front. Microbiol. 8:204. doi: 10.3389/fmicb. 2017.00204

Cardinale, B. J., Duffy, J. E., Gonzalez, A., Hooper, D. U., Perrings, C., Venail, P., et al. (2012). Biodiversity loss and its impact on humanity. Nature 486, 59-67.

Cayuela, H., Gillet, L., Laudelout, A., Besnard, A., Bonnaire, E., Levionnois, P., et al. (2019). Survival cost to relocation does not reduce population self-sustainability in an amphibian. Ecol. Appl. 29:e1909. doi: 10.1002/eap. 1909

Cochran-Biederman, J. L., Wyman, K. E., French, W. E., and Loppnow, G. L. (2015). Identifying correlates of success and failure of native freshwater fish reintroductions. Conserv. Biol. 29, 175-186.

Dincǎ, V., Bálint, Z., Vodǎ, R., Dapporto, L., Hebert, P. D. N., and Vila, R. (2018). Use of genetic, climatic, and microbiological data to inform reintroduction of a regionally extinct butterfly. Conserv. Biol. 32, 828-837.

Du, H. (2014). Conservation Aquaculture of Dabry's Sturgeon Acipenser dabryanus: Biological, Ethological and Physi-ecological Fitness of the Juveniles in Environmental Enriched Rearing Tanks. Wuhan: Huazhong Agriculture University.

Gajardo, K., Jaramillo-Torres, A., Kortner, T. M., Merrifield, D. L., Tinsley, J., Bakke, A. M., et al. (2017). Alternative protein sources in the diet modulate microbiota and functionality in the distal intestine of atlantic salmon (Salmo salar). Appl. Environ. Microbiol. 83, e2615-e2616.

Haase, P., and Pilotto, F. (2019). A method for the reintroduction of entire benthic invertebrate communities in formerly degraded streams. Limnologica 77:125689. doi: 10.1016/j.limno.2019.125689

Hooper, D. U., Adair, E. C., Cardinale, B. J., Byrnes, J., Hungate, B. A., and Matulich, K. L. (2012). A global synthesis reveals biodiversity loss as a major driver of ecosystem change. Nature 486, 105-108.

International Union for Conservation of Nature [IUCN] and Species Survival Commission [SSC]. (2013). Guidelines for Reintroductions and Other Conservation Translocations. Gland: IUCN.

Jourdan, J., Plath, M., Tonkin, J. D., Ceylan, M., Dumeler, A. C., Gellert, G., et al. (2019). Reintroduction of freshwater macroinvertebrates: challenges and opportunities. Biol. Rev. Camb. Philos. Soc. 94, 368-387.

Jule, K. R., Leaver, L. A., and Lea, S. E. G. (2008). The effects of captive experience on reintroduction survival in carnivores: a review and analysis. Biol. Conserv. 141, 355-363.

Kemp, L., and Roshier, D. (2016). "A trial of release protocols for re-introduction of the bush stone-curlew to southern Australia," in Global Re-introduction Perspectives: 2016 Case-studies from around the globe, ed. P. S. Soorae (Abu Dhabi: IUCN/SSC Re-introduction Specialist Group \& Environment Agency), 117-122.

Letty, J., Marchandeau, S., and Aubineau, J. (2007). Problems encountered by individuals in animal translocations: lessons from field studies. ÉcoScience 14, $420-431$.

Li, X., Zhou, L., Yu, Y., Ni, J., Xu, W., and Yan, Q. (2017). Composition of gut microbiota in the gibel carp (Carassius auratus gibelio) varies with host development. Microb. Ecol. 74, 239-249.

Liu, H., Han, M., Li, S. C., Tan, G., Sun, S., Hu, Z., et al. (2019). Resilience of human gut microbial communities for the long stay with multiple dietary shifts. Gut 68 , 2254-2255. doi: 10.1136/gutjnl-2018-317298

Mace, G. M., Barrett, M., Burgess, N. D., Cornell, S. E., Freeman, R., Grooten, M., et al. (2018). Aiming higher to bend the curve of biodiversity loss. Nat. Sustain. 1, 448-451.

Murgier, J., Everaerts, C., Farine, J., and Ferveur, J. (2019). Live yeast in juvenile diet induces species-specific effects on Drosophila adult behaviour and fitness. Sci. Rep. 9, 8812-8873.

Oliver, T. H., Isaac, N., August, T. A., Woodcock, B. A., Roy, D. B., and Bullock, J. M. (2015). Declining resilience of ecosystem functions under biodiversity loss. Nat. Commun. 6:10122.
Patnode, M. L., Beller, Z. W., Han, N. D., Cheng, J., Peters, S. L., and Terrapon, N. (2019). Interspecies competition impacts targeted manipulation of human gut bacteria by fiber-derived glycans. Cell 179, 59-73.

Pimm, S. L., Jenkins, C. N., Abell, R., Brooks, T. M., Gittleman, J. L., and Joppa, L. N. (2014). The biodiversity of species and their rates of extinction, distribution, and protection. Science 344:1246752.

Sarrazin, F., and Legendre, S. (2000). Demographic approach to releasing adults versus young in reintroductions. Conserv. Biol. 14, 488-500.

Smith, C. C., Snowberg, L. K., Caporaso, J. G., Knight, R., and Bolnick, D. I. (2015). Dietary input of microbes and host genetic variation shape among-population differences in stickleback gut microbiota. ISME J. 9:2515.

Svåsand, T., Kristiansen, T. S., Pedersen, T., Salvanes, A., Engelsen, R., Nævdal, G., et al. (2000). The enhancement of cod stocks. Fish Fish. 1, 173-205.

Tavecchia, G., Viedma, C., Bartolome, M., Antonio, J., Gomez, J., and Oro, D. (2009). Maximizing re-introduction success: assessing the immediate cost of release in a threatened waterfowl. Biol. Conserv. 142, 3005-3012.

Taylor, G., Canessa, S., Clarke, R. H., Ingwersen, D., Armstrong, D. P., Seddon, P. J., et al. (2017). Is reintroduction biology an effective applied science? Trends Ecol. Evol. 32, 873-880.

Teitelbaum, C. S., Converse, S. J., and Mueller, T. (2019). The importance of early life experience and animal cultures in reintroductions. Conserv. Lett. 12:e12599.

Tetzlaff, S. J., Sperry, J. H., and DeGregorio, B. A. (2019). Effects of antipredator training, environmental enrichment, and soft release on wildlife translocations: a review and meta-analysis. Biol. Conserv. 236, 324-331.

Todd, C. R., and Lintermans, M. (2015). Who do you move? A stochastic population model to guide translocation strategies for an endangered freshwater fish in south-eastern Australia. Ecol. Modell. 311, 63-72.

Whiteside, M. A., Sage, R., Madden, J. R., and Börger, L. (2015). Diet complexity in early life affects survival in released pheasants by altering foraging efficiency, food choice, handling skills and gut morphology. J. Anim. Ecol. 84, 1480-1489.

Wilkes-Walburn, J., Wemheuer, B., Thomas, T., Copeland, E., O'Connor, W., Booth, M., et al. (2019). Diet and diet-associated bacteria shape early microbiome development in Yellowtail Kingfish (Seriola lalandi). Microb. Biotechnol. 12, 275-288.

Wong, A. C., Wang, Q., Morimoto, J., Senior, A. M., Lihoreau, M., Neely, G. G., et al. (2017). Gut microbiota modifies olfactory-guided microbial preferences and foraging decisions in Drosophila. Curr. Biol. 27, 2397-2404.

Wu, J. M., Wei, Q. W., Du, H., Wang, C. Y., and Zhang, H. (2014). Initial evaluation of the release programme for Dabry's sturgeon (Acipenser dabryanus Duméril, 1868) in the upper Yangtze River. J. Appl. Ichthyol. 30, 1423-1427.

Yang, B., Ye, C., Yan, B., He, X., and Xing, K. (2019). Assessing the influence of dietary history on gut microbiota. Curr. Microbiol. 76, 237-247.

Yang, Z., Gu, X., Nie, Y., Huang, F., Huang, Y., Dai, Q., et al. (2018). Reintroduction of the giant panda into the wild: a good start suggests a bright future. Biol. Conserv. 217, 181-186.

Yuval, B. (2017). Symbiosis: gut bacteria manipulate host behaviour. Curr. Biol. 27, R746-R747.

Zhang, H., Wei, Q. W., Du, H., and Li, L. X. (2011). Present status and risk for extinction of the Dabry's sturgeon (Acipenser dabryanus) in the Yangtze River watershed: a concern for intensified rehabilitation needs. J. Appl. Ichthyol. 27, $181-185$.

Conflict of Interest: The authors declare that the research was conducted in the absence of any commercial or financial relationships that could be construed as a potential conflict of interest.

Copyright (c) 2020 Yang, Leng, Du, Luo, Wu and Wei. This is an open-access article distributed under the terms of the Creative Commons Attribution License (CC BY). The use, distribution or reproduction in other forums is permitted, provided the original author(s) and the copyright owner(s) are credited and that the original publication in this journal is cited, in accordance with accepted academic practice. No use, distribution or reproduction is permitted which does not comply with these terms. 